\title{
Alkali Metal Handling Practices at NASA MSFC
}

\author{
Patrick G. Salvail and Robert R. Carter \\ NASA Marshall Space Flight Center, Huntsville, AL 35812 \\ (256) 544-1818,FAX:-5877,pat.salvail@msfc.nasa.gov
}

\begin{abstract}
NASA Marshall Space Flight Center (MSFC) is NASA's principle propulsion development center. Research and development is coordinated and carried out on not only the existing transportation systems, but also those that may be flown in the near future. Heat pipe cooled fast fission cores are among several concepts being considered for the Nuclear Systems Initiative. Marshall Space Flight Center has developed a capability to handle high-purity alkali metals for use in heat pipes or liquid metal heat transfer loops. This capability is a low budget prototype of an alkali metal handling system that would allow the production of flight qualified heat pipe modules or alkali metal loops. The processing approach used to introduce pure alkali metal into heat pipe modules and other test articles are described in this paper.
\end{abstract}

\section{INTRODUCTION}

As part of the Nuclear Systems Initiative NASA Marshall Space Flight Center is involved in the development of the SAFE ( Safe Affordable Fission Engine) primary heat transport system prototype. The nominal SAFE-100 core consists of 61 sodium heat pipe modules that collectively transfer $100 \mathrm{~kW}$, from the core to a heat pipe-to-gas heat exchanger. These heat pipes operate isothermally at $973 \mathrm{~K}$. Enthalpy from the hot gas exiting the heat exchanger is converted to mechanical work through a dynamic power conversion technique such as a gas turbine or Stirling cycle. The mechanical system is coupled to an alternator that is used to produce payload electrical power or ion thrust.

As a part of the SAFE-100 effort NASA Marshall has developed an in-house alkali metal handling capability. This capability allows test articles to be charged and their contents evaluated in a controlled environment. Figure 1 shows a picture of the alkali metal handling apparatus that is located at NASA Marshall Space Flight Center's main manufacturing shop Building 4705. The building is a high bay manufacturing and research and development area that provides in-house fabrication services at the Center. This shop has machining, welding, cleaning and heattreating facilities. The alkali metal handling system is modular and flexible to accommodate small samples and multiple heat pipe modules. It may be modified to allow the fill of modules having arbitrary length or even a preassembled core. This equipment is not strictly limited to heat pipes it may also be used to support the development of other candidate systems such as potassium or lithium liquid metal heat transfer loops.

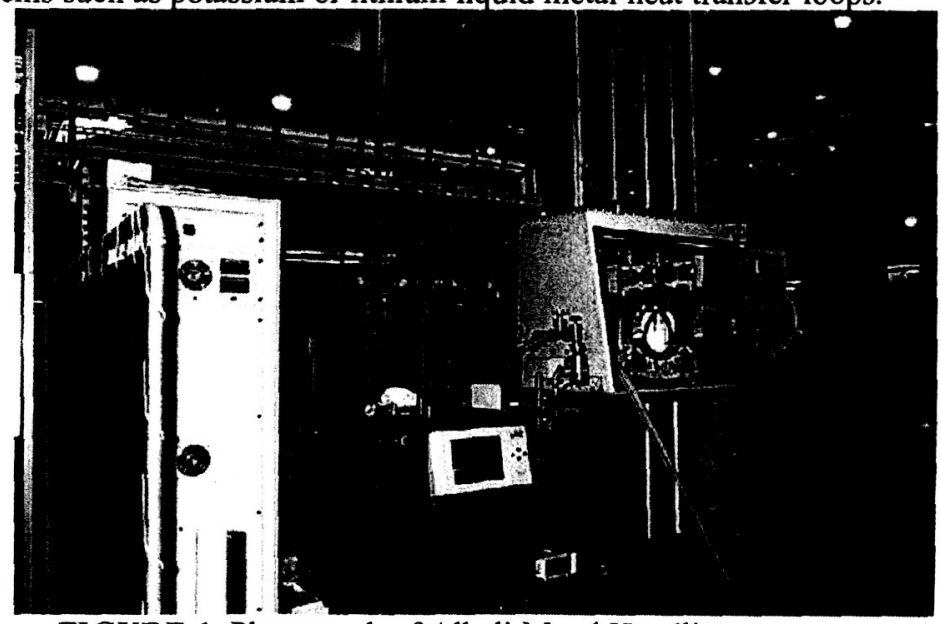

FIGURE 1. Photograph of Alkali Metal Handling Apparatus. 


\section{GLOVE BOX SYSTEM FEATURES}

The alkali metal fill system allows safe repeatable charging of high temperature heat pipes with pure alkali metals. Safe handling procedures were established from the onset of the development effort. Alkali metals, besides being excellent heat transfer mediums, are powerful reducing agents. They can auto-ignite when in air above room temperature and react vigorously with water. Using the experience of Los Alamos National Laboratory (LANL), MSFC introduced safe alkali metal handling practices into the manufacturing cycle. Hazardous materials controls, storage, and waste disposal procedures were established. Each operator is thoroughly briefed on the characteristics of alkali metals and provided with personal protection equipment.

An inert gas glove box with oxygen, moisture, and nitrogen purifiers provides the basis for this flexible handling system. A Nexus glove box system manufactured by Vacuum Atmosphere Corporation, Hawthorne, California produces a pure inert gas environment for the fill operation. The system is made up of the glove box and antechambers, gas purifying systems, oxygen and nitrogen analyzers. The glove box is a hermetically sealed stainless steel chamber, with a full view window. It has two glove ports plus welding, thermocouple, gas, and electrical feed-through. The glove box interior has separate gas and vacuum manifolds. This glove box also has main and mini antechambers located on the right side of the box for the introduction of tools and samples. Three long antechambers, located on top of the glove box, allow placement of heat pipe modules into the box. Up to three heat-pipe modules can be installed into the glove box at one time. Through out operation the system is monitored by the instrumentation unit, which accompanied the glove box system. It controls the Dri Train and Ni Train units using feedback signals from the oxygen and nitrogen analyzers. A four-keyed menu-driven software guides the glove box operator through system operation. This instrumentation system allows for the monitoring and control of glove box activities from a single location.

A National Instruments Lab-VIEW computer data acquisition package monitors and controls the alkali metal distribution system heaters, vacuum levels, and temperatures. Lab-VIEW also monitors the glove box regeneration and purification operations along with the circulation blower speed and the oxygen and moisture levels. This software, along with a few back-up thermal readouts, offers failsafe operation that also provides a record of the fill process. Utilities for the system are accessed from the rear of the glove box. The electrical supply is a 208/110VAC, single-phase 100-A service. Besides providing power to operate each of the glove box components, the outlets provide for the heaters, data acquisition and control computer, scale, thermocouple readouts, and the vacuum pumps that support the system. Compressed air for pneumatic actuators on the nitrogen purification unit is supplied at the location by means of shop distribution. A filter and dryer have been integrated into the line. High purity argon gas is supplied in close proximity to the glove box.

\section{OPERATING PROCEDURES FOR SAFE 100 FILL}

The fill system can handle sodium and potassium in solid, liquid, or vapor forms. The Nexus system contains a nitrogen purifier that also permits high purity lithium to be transferred into modules in solid or liquid forms. The fill system is now configured to introduce pre-distilled liquid sodium into three modules at a time. The machine can fill up to 100 modules before the sodium stock needs replenishment. The SAFE 100 sodium heat pipe module fill steps are shown schematically in Figure 2. The heat pipe modules are metal cylinders approximately 1.2-m long and 1.59-cm OD longitudinally hot iso-statically pressed to similar though shorter fuel tubes. Sodium is introduced at the module condenser end through a $6-\mathrm{mm}$ OD tube fill tube. Each module is charged with $\sim 35 \mathrm{~g}$ of sodium.

A sodium reservoir, located atop the glove box, is connected to three ports within the box. Thermocouples, spot welded to sodium line surfaces, read temperature of the sodium reservoir, sodium supply manifold, the three sodium feeds into the box, and the three sodium lines on the inside of the box. Thermocouple spares are attached in hard to reach locations. The reservoir and supply lines are wrapped in electrical resistance heater tapes that keep them above the sodium melt point during transfer. The fill apparatus thermocouples and heater tapes are connected to the National Instruments data acquisition and control system. Separate virtual instrument software programs are written for each phase of operation. The measured sodium line temperatures are sent to the control system that actuates relays that control the electrical resistance heaters keeping section near the desired set point.

Los Alamos heat pipe fill procedures were modified to allow the fill of modules in a semi-production manner that is both time and cost effective. A three-heat-pipe module fill cycle lasts three days and requires a fill team consisting of two contractors to monitor the gas purification, heating, and data acquisition systems. 


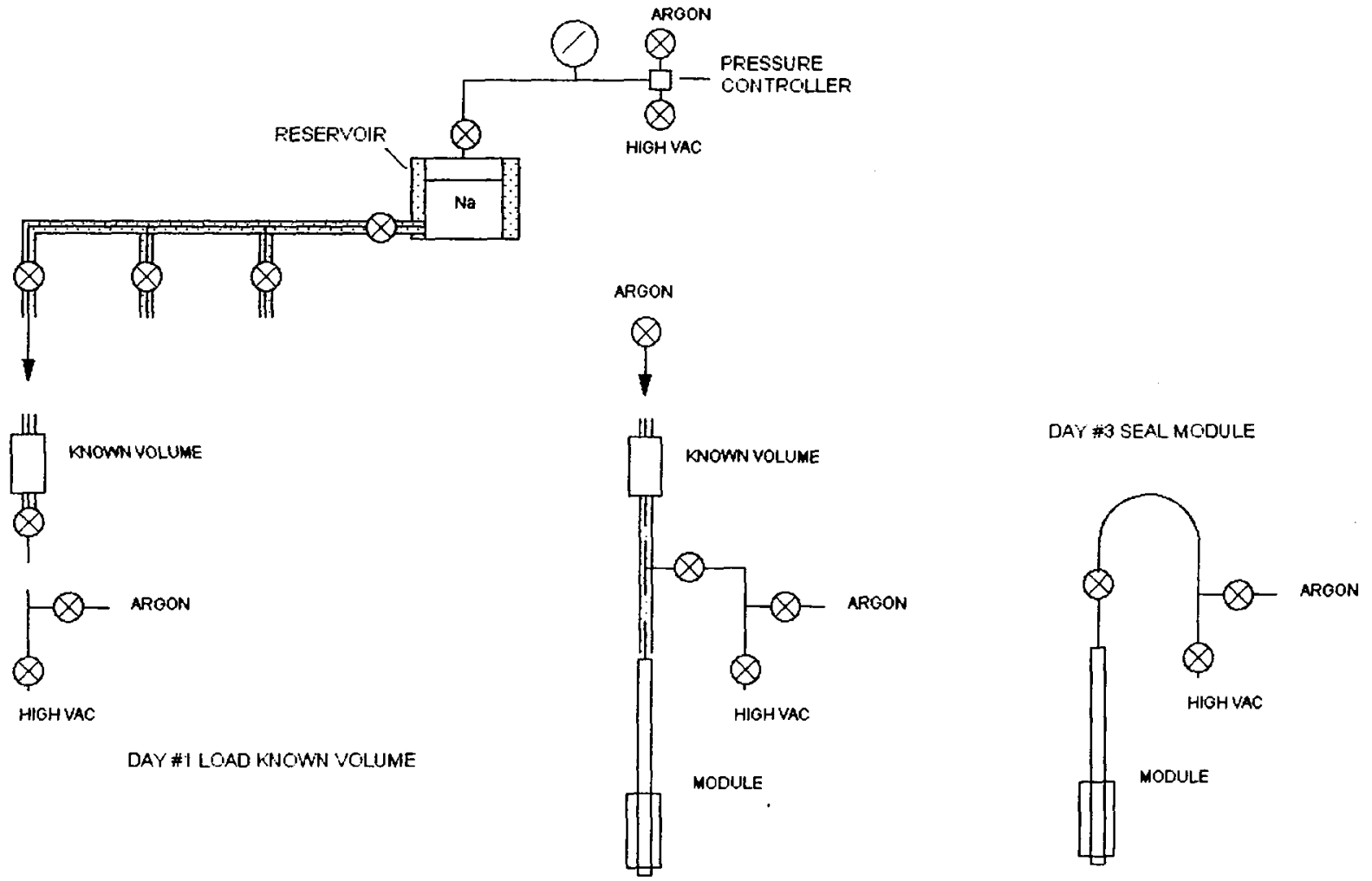

DAY \#2 FILL OF MODULE

FIGURE 2. Schematic Diagram of Alkali Metal Fill Apparatus Sodium Supply Procedure.

A schematic showing the layout for the first day of operation is shown on the left side of Figure 2 . Known volumes are attached on one side to each of the three sodium manifold ports. On the opposing end the known volumes are attached to vacuum/argon supply lines. The known volume canisters are then evacuated. The vacuum manifold is evacuated to $10^{-6}$ torr and then back filled with argon to dilute residual oxygen and nitrogen. When a predetermined purity is achieved the vacuum/argon valves are shut. The transfer lines from the reservoir to the know volumes are brought to $\sim 120^{\circ} \mathrm{C}$. Three heaters inside the box wells use the minimum indicated temperatures as the control signal. The coldest sodium manifold temperature also controls the heater output inside the glove box. The National Instruments system sends a 0-10 VDC signals to a proportional pressure controller to pressurize the sodium supply reservoir. The valve connecting the sodium manifold to each known volume is opened and each canister is filled with sodium. The routine shifts to the module fill.

Power is cut to the manifold heaters, the system is allowed to cool, and heat pipe modules are introduced into the glove box. After an overnight gas purification of the system to about $1 \mathrm{ppm}$ of $\mathrm{O}_{2}$, the known volumes are disconnected from the sodium manifold. The known volumes are weighed to confirm that sodium completely filled each. The known volumes are then connected on one end to a carrier gas manifold (middle depiction on Figure 2). The other end of the known volume branches to the vacuum/argon supply line and the heat pipe module. The module is cleansed by successive argon back fill and evacuation. The valve connecting the known volume-module line is then closed, the sodium melted, and argon carrier gas pushes sodium into the module. Another overnight cool down and purification is carried out.

On the third day, the heat pipe modules are disconnected from the known volumes. A valve is attached to the fill stem of each module (depicted on the right side of Figure 2). The module is then pumped down under high vacuum and the fill stem valve is closed. The modules are removed from the glove box system, weighed to confirm their fill, and crimped at the fill stem. They are then heated to bring the sodium to the crimped end, and returned to the glove box for cutting and welding of the fill stem. After TIG welding of the fill stem inside the glove box the pipe is placed in a vacuum tube furnace at $700^{\circ} \mathrm{C}$ for a 48 -hour wet-in process. 


\section{WORKING FLUD SOURCE}

Alkali metal systems such as heat pipes and liquid metal loops are sensitive to the concentration of impurities in the working fluid and wall especially calcium, carbon, silicon, nitrogen, and oxygen. Processing procedures have evolved and refined to keep non-metallic impurities within these systems to acceptable levels. These processing procedures also eliminate outgas products. The alkali metal source used for the SAFE 100 modules is NIAPURE sodium provided by DuPont. It was produced by the Downs electrolytic process and is among the purest commercially available stock. Table 1 compares impurity concentrations quoted by DuPont for various elements in NIAPURE to that of standard sodium. Oxygen concentration measurements were made by neutron activation analysis. To remove trace non-metallic impurities such as carbon, calcium, nitrogen, oxygen, phosphorous, and sulfur this sodium was further purified by vacuum distillation. An inline filter assures that final oxygen concentrations in the sodium supplied to each module are under $10 \mathrm{ppm}$.

The distilled sodium was provided to NASA MSFC inside stainless steel containers that were backfilled with argon cover gas. Figure 3 shows a schematic of the distillation fill apparatus. The reservoir containers were fabricated in two pieces so that the interior could be readily cleaned and vacuum fired. Once the interior surfaces were vacuum fired at $400^{\circ} \mathrm{C}$, the two pieces were electron beam welded together, and a final vacuum fire step was repeated. Charging the reservoirs with sodium consists of five separate operations: (1) the movement of NIAPURE sodium from a storage container to a loading pot of a known volume; (2) the transfer of sodium from the known volume to the distillation pot; (3) the vaporization of sodium in the distillation pot and condensation of the sodium in a line that allows the distillate to gravity-feed into the reservoir; (4) backfilling the reservoir voids with high purity argon, and (5) the attachment valves and end caps to the reservoirs under an inert gas atmosphere.

TABLE 1. Impurity Concentrations in NIAPURE Sodium Given by DuPont.

\begin{tabular}{|c|c|c|c|}
\hline Symbol & Element & $\begin{array}{c}\text { RegularGrade } \\
\text { (ppm) }\end{array}$ & $\begin{array}{c}\text { NIAPUREGrade } \\
\text { (ppm) }\end{array}$ \\
\hline$\overline{\mathrm{Ag}}$ & Silver & $<28$ & 14 \\
\hline $\mathrm{Al}$ & Aluminum & 4 & 1.6 \\
\hline $\mathrm{Ba}$ & Barium & 2 & 2 \\
\hline $\mathrm{Be}$ & Beryllium & $<0.5$ & $<1$ \\
\hline B & Boron & 2 & $<2$ \\
\hline $\mathrm{Ca}$ & Calcium & 330 & 4 \\
\hline $\mathrm{C}$ & Carbon & ND & 1.7 \\
\hline $\mathrm{Cl}$ & Chlorine & 30 & $<5$ \\
\hline $\mathrm{Cr}$ & Chromium & $<5$ & 0.8 \\
\hline $\mathrm{Cu}$ & Copper & $<18$ & 1.7 \\
\hline $\mathrm{Fe}$ & Iron & 2.4 & 0.9 \\
\hline $\mathrm{K}$ & Potassium & 300 & 300 \\
\hline $\mathrm{Li}$ & Lithium & $<10$ & $<1$ \\
\hline $\mathrm{Mg}$ & Magnesium & 9 & 4 \\
\hline $\mathrm{Mn}$ & Manganese & $<1.2$ & 0.4 \\
\hline Mo & Molybdenum & $<6$ & 0.4 \\
\hline $\mathrm{N}$ & Nitrogen & ND & $<3$ \\
\hline $\mathrm{Ni}$ & Nickel & $<9$ & 0.9 \\
\hline $\mathbf{P}$ & Phosphorous & $<20$ & 6 \\
\hline $\mathrm{Si}$ & Silicon & 5 & 7 \\
\hline $\mathrm{Sr}$ & Strontium & 21 & $<1.3$ \\
\hline Sn & Tin & $<12$ & $<25$ \\
\hline $\mathrm{S}$ & Sulfur & 10 & 0.4 \\
\hline $\mathrm{Ti}$ & Titanium & 3.6 & $<6$ \\
\hline $\mathrm{V}$ & Vanadium & 8.2 & 0.02 \\
\hline $\mathrm{Zn}$ & Zinc & 30 & $<6.3$ \\
\hline $\mathrm{Zr} / \mathrm{Hf}$ & Zirconium/Hafnium & ND & 2 \\
\hline
\end{tabular}




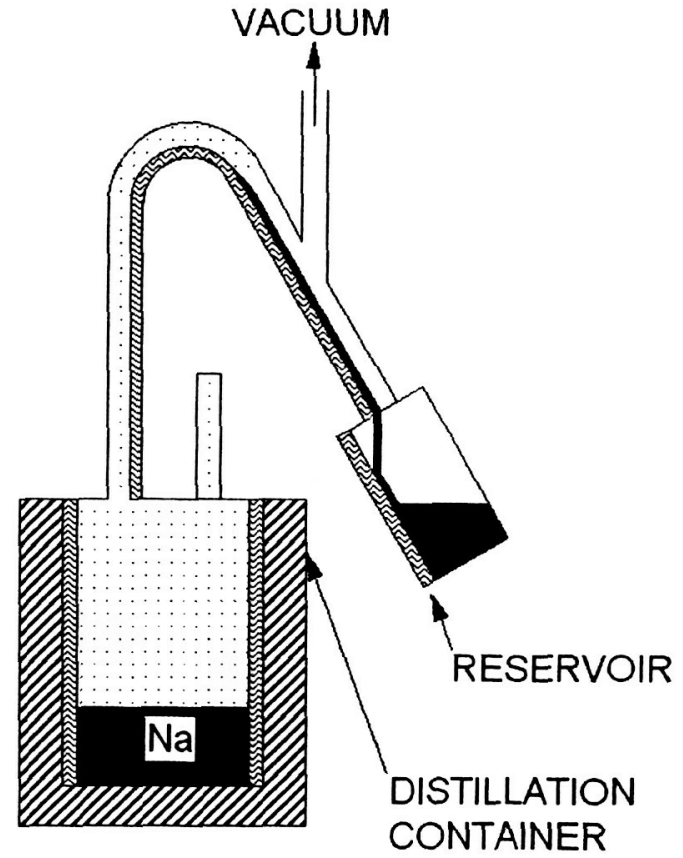

FIGURE 3. Distillation Apparatus Used to Fill Sodium Reservoirs.

\begin{abstract}
SUMMARY
The alkali metal handling capability described in this paper gives MSFC the ability to fill and examine components used in alkali metal heat pipes and heat transfer loops. It also serves as a prototype alkali metal system for a flight program. To keep fill prototype development cost below $\$ 0.5 \mathrm{M}$, sodium purification was done by bulk vacuum distillation. The manufacture of alkali metal heat pipes or a liquid metal loop for a flight system would justify the development of a more elaborate handling capability. An advanced fill system for flight qualified units would vacuum distill alkali metal into individual heat pipe modules and might be made to fill modules for an entire core simultaneously. Detailed assays of non-metallic and gas residual impurity levels might also be performed in situ. A stricter fabrication and quality control regimen would be justified for space flight qualified components. Fabrication and quality control procedures could be checked using accelerated life tests on sample modules at elevated temperature and various impurity levels. An advanced alkali metal handling system could also be used to conduct chemical and metallographic examinations on sample modules during and after accelerated life test for evidence of corrosive attack and to determine impurity distributions.
\end{abstract}

\title{
ACKNOWLEDGMENT
}

This work was supported by NASA Marshall Space Flight Center, Huntsville, AL. 